\title{
Prueba de alergia a la penicilina. ¿Necesaria o innecesaria?
}

\section{Penicillin allergy test. Necessary or unnecessary?}

\author{
Daniel Ramón Gutiérrez Rodríguez ${ }^{1}$, Carlos Alberto Ulloa Benitez ${ }^{2}$ \\ ${ }^{1}$ Universidad Católica de Cuenca, Ecuador \\ ${ }^{2}$ Hospital General Calixto García, Cuba \\ *danielgr0205@gmail.com
}

DOI: https://doi.org/10.26871/killkana_salud.v4i2.521

\begin{abstract}
Resumen
Contexto: La frecuencia de las reacciones a los medicamentos aumenta con la exposición repetida a medicamentos, y es más común en adultos que en niños. Las reacciones de hipersensibilidad tienen características diferentes de las reacciones que no responden a los mecanismos inmunológicos, están relacionadas con los efectos farmacológicos conocidos del medicamento y, por lo tanto, son predecibles. En la actualidad, esto le pasa aproximadamente al $10 \%$ de la población. Objetivo: Analizar a través de fuentes bibliográficas científicas los efectos de la penicilina en diferentes pacientes. Metodología: Se empleó el análisis documental a diferentes fuentes científicas que hacen referencia a pacientes que han mostrado alergias a la penicilina. Resultados: Las historias de bajo riesgo incluyen con síntomas no alérgicos aislados, como síntomas gastrointestinales, o pacientes con antecedentes familiares de alergia a la penicilina. El sexo femenino ha sido identificado como un factor de riesgo en adultos para la alergia autoinformada a la penicilina y la alergia confirmada. Tampoco parece haber una relación importante entre la atopia y la incidencia de alergia a la penicilina. Un resultado positivo indica alergia, siempre que se pruebe que las concentraciones utilizadas no son irritantes. Un resultado negativo no excluye la alergia a la medicación. La prueba cutánea para excluir o confirmar un mecanismo mediado por $\operatorname{IgE}$ se considera positiva cuando se produce una erupción o erupción cutánea y eritema después de la inyección intradérmica. Conclusiones: El valor predictivo de las pruebas cutáneas con medicamentos, principalmente penicilina, es incierto. Es muy común que esta prueba se realice en personas que no tienen antecedentes de reacción a la penicilina, lo que no tiene sentido, ya que en un individuo sin antecedentes médicos que sugiera hipersensibilidad, la posibilidad de presentar una reacción grave por este medicamento es muy poco probable. $(0,004 \%$ a $0,015 \%)$.
\end{abstract}

Palabras clave: Hipersensibilidad, penicilina, pruebas cutáneas .

\begin{abstract}
Context: Drug reactions frequency increases with repeated exposure to medications, and it is more common in adults than in children. Hypersensitivity reactions have different characteristics from those not responding to immunological mechanisms. They are related to the known pharmacological effects of the drug; therefore, they are predictable. Currently, this happens to approximately 10\% of the population. Objective: To analyse the effects of penicillin in different patients, through scientific literature sources. Methodology: The documentary analysis of different scientific sources referring to patients who presented allergies to penicillin, was carried out. Results: Low-risk medical records include isolated nonallergic symptoms, such as gastrointestinal distress, or patients having penicillin allergies family history. The female sex has been identified as a risk factor in adults for both self-reported and confirmed penicillin allergy. There appears to be no significant relationship between atopy and the incidence of penicillin allergy. A positive result is an indicator of allergy, provided that the concentrations used are proven not to be irritating. A negative result does not exclude medication allergy. The skin testing for excluding or confirming an IgE-mediated mechanism is considered positive when a rash, or skin rash and erythema, appears after intradermal injection. Conclusions: The predictive value of skin testing with drugs, mainly penicillin, is uncertain. It is ubiquitous for this test to be performed on people with no history of reaction to penicillin. This procedure makes no sense, since in an individual with no medical history of hypersensitivity, the possibility of having a serious reaction to this drug is improbable. $(0,004 \%$ a $0,015 \%)$.
\end{abstract}

Keywords: Hypersensitivity, penicillin, skin tests.

\section{Introducción}

La frecuencia de las reacciones a medicamentos aumenta con la exposición repetida a fármacos, es más frecuente en adultos que en niños. La mayoría de estas reacciones no son alérgicas y tienen relación con las acciones farmacológicas del medicamento. Una reacción de hipersensibilidad debe tener unas características independientes de las acciones propias del medicamento. ${ }^{1}$ 
Las reacciones alérgicas a medicamentos se engloban en reacciones de tipo A son las más frecuentes y corresponderían al 85-90\% del total de las reacciones adversas, son de tipo previsibles y guardan relación con el mecanismo de acción del medicamento, su aparición es posible en todos los individuos tratados, en general son reacciones de características leves, dependientes de la dosis y son las más frecuentes. Las de tipo B (no previsibles) y se deben a respuestas anómalas de una pequeña parte de la población, no están relacionadas con la dosis y son menos frecuentes, pueden deberse a intolerancia, idiosincrasia, las pseudo alérgicas y las de hipersensibilidad, resultante de una respuesta inmune; estas últimas son las reacciones alérgicas a medicamentos. $^{2}$

La prevalencia real de la alergia a medicamentos es mal conocida, la mayoría de autores aceptan porcentajes entre el 6 y el $10 \%$ de las reacciones adversas a fármacos. ${ }^{1} \mathrm{La}$ mayoría de estudios realizados se han llevado a cabo en medio hospitalario, lo que ya supone un sesgo importante. En edad pediátrica, se supone baja, menor que en el adulto.

Las reacciones por hipersensibilidad tienen características diferentes de las reacciones que no responden a mecanismos inmunológicos, se relacionan con los efectos farmacológicos conocidos del fármaco y por ello son previsibles, por lo tanto, las inmunológicamente mediadas:

- Son distintas de las acciones farmacológicas conocidas del fármaco.

- Existe un contacto previo con el fármaco implicado (continuo o intermitente) que causa la sensibilización del paciente.

- Sus manifestaciones clínicas deben concordar con las propias de las reacciones alérgicas conocidas.

- $\quad$ Son recurrentes ante nuevas exposiciones al alérgeno.

- La reacción debe mejorar al retirar el fármaco.

- La reacción debe ser reproducible con dosis mínimas de fármaco.

- Es posible la reactividad cruzada con fármacos de estructura similar. ${ }^{3}$

La clasificación de alergia a medicamentos cronológicamente (según la cadencia de aparición de los síntomas). Hasta hace unos años se siguió usando la clasificación que Levine presentó para clasificar las reacciones a penicilina. ${ }^{4}$ Ordenaba las reacciones basándose en el momento de la aparición de los síntomas.

- Inmediatas: 1-60 minutos tras la administración del fármaco.

- Aceleradas: 1-72 horas tras la administración del fármaco.

- Tardías: a partir de las 72 horas tras la administración del fármaco.

La exposición repetida a antibacterianos, por ejemplo en condiciones médicas que requieren un uso antibacteriano frecuente, como la fibrosis quística, se reconoce como un factor de riesgo clínico para la alergia a la penicilina. ${ }^{5}$

Ahora bien, que se dirá pues de los antibióticos $\beta$ lactámicos, estos se encuentran entre los antibióticos más seguros y efectivos. Muchos pacientes reportan alergias a estos medicamentos que limitan su uso, lo que da como resultado el uso de antibióticos de amplio espectro que aumentan el riesgo de resistencia a los antimicrobianos y eventos adversos. ${ }^{6}$

Actualmente alrededor del $10 \%$ de la población de EE.UU. ha informado de alergias a la penicilina, un agente lactámico $\beta$, con tasas más altas registradas por pacientes de mayor edad y hospitalizados. Aunque muchos pacientes informan que son alérgicos a la penicilina, la hipersensibilidad a la penicilina mediada por IgE o por linfocitos $\mathrm{T}$ clínicamente significativa es poco frecuente $(<5 \%)$. Actualmente, la tasa de alergias a la penicilina mediada por IgE está disminuyendo, posiblemente debido a un uso disminuido de las penicilinas parenterales, y porque las reacciones anafilácticas graves a la amoxicilina oral son raras. La alergia a la penicilina mediada por $\operatorname{IgE}$ disminuye con el tiempo y el $80 \%$ de los pacientes se vuelven tolerantes después de una década. La reactividad cruzada entre penicilina y cefalosporinas se produce en aproximadamente el $2 \%$ de los casos, menos del $8 \%$ reportado anteriormente. Algunos enfermos tienen un historial médico que sugiere que tienen un bajo riesgo de desarrollar una reacción alérgica a la penicilina. Las historias de bajo riesgo incluyen enfermos con síntomas no alérgicos aislados, como síntomas gastrointestinales, o pacientes con antecedentes familiares de alergia a la penicilina, síntomas de prurito sin erupción o reacciones remotas (>10 años) desconocidas sin características que sugieran una IgE reacción mediada. ${ }^{6}$

El sexo femenino ha sido identificado como un factor de riesgo en adultos tanto para la alergia a la penicilina autoinformada como para la alergia confirmada, ${ }^{7,8}$ posiblemente relacionado con un mayor uso antibacterianos en mujeres. ${ }^{7}$ La prevalencia de la alergia a la penicilina parece aumentar con la edad y podría explicarse en parte por mayores tasas de exposición antibacteriana en los grupos de mayor edad. ${ }^{8}$

También se ha encontrado que una historia familiar de alergia a la penicilina está asociada con la alergia a la penicilina autoinformada, sin embargo, si hay un componente genético de la alergia a la penicilina es algo que hasta estos momentos no está claro, en otras palabras, no hay un esquema predecible con respecto a la herencia de la alergia a la penicilina. ${ }^{9}$ Por lo tanto, no es necesario que el paciente evite la penicilina porque un miembro de su familia sea alérgico a la misma o a fármacos de la familia de las penicilinas. ${ }^{10}$ Tampoco parece haber una relación importante entre la atopía y la incidencia de alergia a la penicilina. 5 Sin embargo, el British National Formulary aconseja que las personas con alergias atópicas podrían estar en mayor riesgo de reacciones anafilácticas a las penicilinas. ${ }^{9}$ Hasta la actualidad no se han identificado factores de riesgo específicos en niños. ${ }^{5}$ En un estudio de niños (edad media de 3,5 años, rango de 6 meses a 14,5 años) en quienes se había reportado erupción urticarina tardía o erupción maculopapular después de la administración de penicilina, la erupción recidivó en sólo $6,8 \%$ en el los test 
de provocación. ${ }^{11}$ Las infecciones virales se detectaron en la mayoría de los niños con un resultado del re-test negativo y se ha sugerido que muchas erupciones atribuidas a la alergia de la penicilina podrían ser de origen viral.

Un historial de riesgo moderado incluye urticaria u otras erupciones pruríticas y reacciones con características de las reacciones mediadas por IgE (anafilaxia). Un historial de alto riesgo incluye pacientes que han tenido anafilaxis, pruebas cutáneas positivas con penicilina, reacciones recurrentes a la penicilina o hipersensibilidad a múltiples antibióticos $\beta$-lactámicos. Los objetivos de la administración antimicrobiana se ven perjudicados cuando la alergia reportada a la penicilina conduce al uso de antibióticos de amplio espectro que aumentan el riesgo de resistencia antimicrobiana, incluido un mayor riesgo de Staphylococcus aureus resistente a la meticilina y Enterococcus resistente a la vancomicina. Los agentes antimicrobianos de amplio espectro también aumentan el riesgo de desarrollar una infección por Clostridium difficile. El reto continuo con amoxicilina es apropiado para pacientes con antecedentes de alergia de bajo riesgo. ${ }^{6}$

La penicilina es una excepción y se dispone de metabolitos y complejos metabolito/ proteína transportadora para diagnóstico, pero esto no ocurre con otros fármacos por lo que se usa el fármaco en forma original (no metabolizado). Un resultado positivo indica alergia, siempre que esté probado que las concentraciones usadas no son irritantes. Un resultado negativo no excluye alergia al fármaco. ${ }^{3}$ Los pacientes de riesgo moderado pueden ser evaluados con pruebas de penicilina en la piel, que tienen un valor predictivo negativo que supera el $95 \%$ y se acerca al $100 \%$ cuando se combina con el desafío con amoxicilina. Los médicos que realizan la evaluación de la alergia a la penicilina deben identificar qué métodos están respaldados por los recursos disponibles. ${ }^{6}$

Las reacciones alérgicas a la penicilina y a las penicilinas semisintéticas se pueden clasificar como se ha visto en inmediatas (anafilácticas), que se producen en el curso de los 60 minutos siguientes a la administración; aceleradas, cuando comienzan entre 1 y 72 horas después; y tardías, si aparecen después de 72 horas de administrado el fármaco. La reacción anafiláctica representa la principal amenaza para la vida. Las reacciones aceleradas consisten por lo general en manifestaciones cutáneas o enfermedad del suero, pero los trastornos letales son raros. Las reacciones tardías incluyen la dermatitis exfoliativa, el síndrome de StevensJohnson, la enfermedad del suero y la nefritis intersticial, dermatitis de contacto, necrólisis epidémica toxica, exantema fijo pigmentario, erupciones exantemáticas maculopapulares, postulosis aguda generalizada exantemática, aunque se han identificado otras manifestaciones, como postulosis necrotizante y eritema polimorfo.

La prueba cutánea para excluir o confirmar un mecanismo mediado por la IgE se considera positiva cuando se produce una roncha o habón y eritema después de la inyección intradérmica $(0,02 \mathrm{ml})$ en dilución adecuada.
No obstante, es incierto el valor predictivo de las pruebas cutáneas $^{12}$ a medicamentos principalmente a la penicilina. Cabe notar que después de la recuperación del cuadro clínico ocasionado por una segunda exposición, el proceso se puede precipitar de nuevo en la misma forma con una sola dosis de prueba, aunque se encuentre diluida a la concentración adecuada, y que además la reacción no está relacionada con la magnitud de la dosis o con la cantidad total administrada. ${ }^{13}$

Es de señalar que las pruebas cutáneas deben realizarse solamente para descartar una sospecha clínica, donde el paciente ha estado tomando varios medicamentos $y$ ha ocurrido una reacción, no precisando al medicamento responsable, y en este sentido no está indicado estudiar ningún individuo sin historia de reacción. Tampoco deben realizarse cuatro semanas después de ocurrida la reacción, debido a que se pueden obtener falsos negativos por depleción de mediadores y/o IgE especifica durante la reacción; además después de varios días la determinación de $\operatorname{IgE}$ especifica frente a penicilina disminuye, y se vuelve a niveles indetectables al año de producida la reacción sospechosa si no existen otros estímulos antigénicos, sin embargo hay que ser prudentes a la hora de evaluar la determinación de IgE sérica frente a penicilina u otro medicamento, ya que esta no mide la IgE unida a mastocitos y basófilos, que es la que pone en marcha la reacción alérgica inmediata. ${ }^{2}$ El tipo de prueba cutánea se elegirá según si la reacción ha sido inmediata o tardía. En dependencia del cuadro presentado, pueden presentarse formas mixtas como urticaria y angioedema con aparición de varios días de otra manifestación alérgica; en dicho caso el enfoque diagnostico será más difícil.

Desde hace muchos años se ha utilizado la prueba intradérmica con penicilina $\mathrm{G}$ a dilución de 1/10 000, utilizando siempre un control positivo y uno negativo, para descartar o confirmar la presencia de alergia, ${ }^{14}$ no admite justificación aplicar la penicilina sin diluir, ya que el riesgo de reacción alérgica o irritativa aumenta, no obstante, las dosis moderadas o bajas con intervalos libres sensibilizan más que las dosis elevadas y sin pausas. ${ }^{2}$

Ahora bien se sabe qué, confirmar o descartar el diagnóstico con dicha prueba carece de cualquier valor clínico en la actualidad, de acuerdo con los consensos internacionales ${ }^{14}$ donde la negatividad de la pueba no descarta la posibilidad de una alergia medicamentosa, si la reacción que ocasiono la sospecha de alergia al fármaco puso en peligro la vida del paciente, o podría dejar secuelas, no está indicado realizar pruebas ni intradérmicas ni de parche, por lo tanto esta contraindicado totalmente si la reacción fue una anafilaxia grave, un síndrome de Steven-Jonhson o una necrólisis epidérmica toxica (Síndrome Lyell) . De hecho, las reacciones a las penicilinas pueden presentarse contra diferentes haptenos y es por ellos que realizar una prueba con uno solo de ellos tiene una sensibilidad cercana al $30 \%$. Es muy frecuente que esta prueba se realice apersonas que no tienen historia de reacción a la penicilina, lo cual no tiene 
ningún sentido, pues en un individuo sin historia clínica que sugiera hipersensibilidad la posibilidad de presentar una reacción grave por este fármaco es muy poco probable $(0,004 \%$ al $0,015 \%){ }^{15}$ Tanto en las pruebas cutáneas o en las analíticas para medicamentos, un resultado positivo es muy informativo, pero un resultado negativo se ha de informar muy cautamente ya que la información se debe interpretar como que no se puede detectar anticuerpos de tipo IgE frente al medicamento testado, pero no se puede afirmar su inexistencia. ${ }^{2}$ En términos generales se puede decir que las pruebas cutáneas a Prick e intradérmicas 10. constituyen la mejor herramienta para el diagnóstico de las reacciones inmediatas, pero no para las aceleradas y tardías, las cuales requieran la realización de pruebas de parche con lecturas tardías, por personal entrenado. ${ }^{15}$

\section{Fuente de Financiamiento}

Este estudio es autofinanciado.

\section{Conflicto de Intereses}

No existen conflictos personales, profesionales, financieroso de otro tipo.

\section{Consentimiento Informado}

Los autores cuentan con el consentimiento informado de los pacientes para la investigación, la publicación del caso y sus imágenes.

\section{Referencias Bibliográficas}

1. Cortada Macías J, López Serrano M, Blasco A, Mayorga C, Torres M. Introducción, conceptos generales, epidemiología. Fisiopatología: los fármacos como antígenos. Tratado de alergología Madrid: Ergon. 2007:1297-324.

2. Giner M, Mateos M. Alergia a Farmacos.; 2011.

3. Giner Muñoz MT. Alergia a medicamentos. Conceptos básicos y actitud a seguir por el pediatra. Protocolos diagnosticos pediatria; 2003.

4. Levine BB. Immunologic mechanisms of penicillin allergy: a haptenic model system for the study of allergic diseases of man. New England Journal of Medicine. 1966;275(20):1115-1125.

5. Mirakian R, Leech S, Krishna M, Richter A, Huber P, Farooque S, et al. Management of allergy to penicillins and other beta-lactams. Clinical \& Experimental Allergy. 2015;45(2):300-327.

6. Shenoy ES, Macy E, Rowe T, Blumenthal KG. Evaluation and management of penicillin allergy: a review. Jama. 2019;321(2):188-199.
7. Mota I, Gaspar Â, Chambel M, Piedade S, MoraisAlmeida M. Hypersensitivity to beta-lactam antibiotics: a three-year study. European annals of allergy and clinical immunology. 2016;48(6):212.

8. Park MA, Matesic D, Markus PJ, Li JTC. Female sex as a risk factor for penicillin allergy. Annals of Allergy, Asthma \& Immunology. 2007;99(1):54-58.

9. Macy E, KY TP. Self-reported antibiotic allergy incidence and prevalence: age and sex effects. The American journal of medicine. 2009;122(8):778-e1.

0. Pongdee T. Inmunología, Academia Americana; 2019. Available from: https://www.aaaai.org/conditions-andtreatments/library/allergy-library/sp-penicillin-allergyfaq.

11. Apter AJ, Schelleman H, Walker A, Addya K, Rebbeck T. Clinical and genetic risk factors of self-reported penicillin allergy. Journal of allergy and clinical immunology. 2008;122(1):152-158.

12. Vervloet D. Allergy to muscle relaxants and related compounds. Clinical \& Experimental Allergy. 1985;15(6):501-508.

13. Lazo-Salazar M. Alergia Medicamentosa. Revista Facultad de Ciencias Médicas de la Universidad de Guayaquil. 2012;15(2). Available from: http://www.ug.edu.ec/revistas/Revista_Ciencias_ Medicas/REVISTA_N2_VOL15/RevistaMedicinaVol15_ 2_Rep_farmacologia.pdf.

14. Cardona Villa R, Serrano Reyes C. Alergia a betalactámicos. 1st ed. Garrido Madrid A, editor. Bogotá: Editorial Médica Internacional LTDA; 2010.

15. Torres MJ, Blanca M, Fernandez J, Romano A, De Weck A, Aberer W, et al. Diagnosis of immediate allergic reactions to beta-lactam antibiotics. Allergy. 2003;58(10):961-972.

Recibido: 25 de septiembre de 2019

Aceptado: 12 de mayo de 2020 